and explanations were clearly delusional. However, no aspect of identity-formation was completely unique to psychosis or nonpsychosis diagnostic groups. The identity-formation process, coping strategies, and outlooks can be seen as a framework both for individual therapies and further research.

\section{Service evaluation for services for younger people with dementia in east locality of north Wales}

Asha Dhandapani*, Sathyan Soundararajan

and Sharmi Bhattacharyya

BCUHB

${ }^{\star}$ Corresponding author.

doi: 10.1192/bjo.2021.108

Aims. To evaluate Young-onset dementia (YOD) services in terms of referral, its appropriateness, time to diagnosis and other criteria as per protocol that we have adapted.

Method. Case notes of those under 65 referred to Memory service for cognitive assessment between July 2017 and June 2018 were retrospectively reviewed to look at the time to diagnosis, appropriate referrals, post-diagnostic support, etc.

Result. Compared to the previous evaluation, the number of patients referred to had increased from 47-48/ year earlier to $63 /$ year. Only $1 / 3$ were appropriate referral over the 10 -year period whereas between 2017 and 2018 more than half were appropriate referrals. More than half of them were seen within 12 weeks of referral (35/63 available). Only 132/252 were diagnosed as having some form of dementia in the previous evaluation which was about 13 cases of YOD a year. In contrast, in our new evaluation 19 patients were diagnosed with some form of dementia. Inappropriate referrals had reduced by more than $50 \%$. Appropriateness and timely referral had improved in this time frame.

Conclusion. Dementia is considered 'young onset' when it affects people under 65 years of age. It is also referred to as 'early onset' or 'working age' dementia. However, this is an arbitrary age distinction that is becoming less relevant as increasingly services are realigned to focus on the person and the impact of the condition, not the age. Teaching sessions to educate primary \& secondary care clinicians on appropriateness and timely referrals have helped in improving the care for patients with YOD. Services need to be developed further to be able to diagnose \& support those with YOD. Repeat evaluations every year would help to inform improvement in quality \& appropriateness of referrals.

Service user experience of remote consultations during COVID-19 in an older adult community mental health setting

Darena Dineva*, Sukran Altun, Tahiya Twaha and Juliette Brown

East London NHS Foundation Trust

${ }^{*}$ Corresponding author.

doi: 10.1192/bjo.2021.109

Aims. The COVID-19 pandemic has had a significant effect on our ability to communicate face-to-face with patients freely. Similar to other medical specialities and general practice (1), to continue providing a service for our service users, we employed other means of communication including telephone and video consultations (although face-to-face consultations were available for high clinical concern and/or identified risk). We set out to explore the acceptability of remote consultation for service users of an older adult ( $>65$ years) Community Mental Health Team (CMHT).

Reference: BMJ 2020;371:m3945

Method. A total of 34 service users were selected randomly from the CMHT caseload (9\% of total 372 caseload). 4 clinicians were involved in collecting data between July and October 2020. We used our trust's (East London Foundation Trust) standard questionnaire on patient satisfaction and contacted individuals by telephone to complete the survey.

Result. For both questions of 'I felt listened to by the team' and 'I feel I have been given enough information regarding my care' 17 (50\%) responders 'agreed' with this statement and an additional 13 (38\%) 'strongly agreed' (total of $88 \%$ ). For the statement 'I feel involved in decisions about my care' 16 (47\%) responders 'agreed' and a further 11 (32\%) responders 'strongly agreed' with this statement. The statement 'The professionals involved in my care talk to each other and we all work as a team' had 15 (44\%) responders 'agree' and 13 (38\%) responders 'strongly agree'. When asked 'If you experienced telephone/video sessions, were these helpful?' 31 responders said 'yes'.

Conclusion. Overall most responders agreed or strongly agreed that they felt listened to, were given enough information about their care, felt involved in decisions about their care and that they worked as a team with the professionals involved in their care. $91 \%$ of responders felt that the video/telephone sessions had been helpful. These data have provided reassurance that telemedicine methods were a useful substitute for face-to-face consultations during the early stages of the pandemic. However this was a small scale study. This study cannot tell us about the experience after the initial 6 months of the pandemic, how often it would be optimal for people have face to face reviews, or whether satisfaction with telemedicine altered over a longer period. We were also not able to tell whether the experience varied for those who had less robust and longstanding relationships with their clinicians.

\section{Insomnia management; don't sleep on it}

Maria Donnelly ${ }^{1 \star}$, Nieves Mercadillo ${ }^{2}$ and Stuart Davidson ${ }^{2}$

${ }^{1}$ Warrington and Halton Teaching hospitals and ${ }^{2}$ North West

Boroughs Healthcare NHS Foundation Trust

${ }^{*}$ Corresponding author.

doi: 10.1192/bjo.2021.110

Aims. In this project our aim was to improve patient safety and care by reducing hypnotic prescription medication administration. We also wanted to reduce over-prescribing/unnecessary prescribing which has a negative pharmaceutical impact on the environment and is a huge expenditure issue for the NHS. NICE guidance for Insomnia management states "After consideration of the use of non-pharmacological measures, hypnotic drug therapy is considered appropriate for the management of severe insomnia interfering with normal daily life; it is recommended that hypnotics should be prescribed for short periods of time only, in strict accordance with their licensed indications" Side effects are common with hypnotic usage including, most importantly, the development of tolerance and rebound insomnia.

Method. The interventions we implemented included the development of an educational presentation about insomnia, the development of an "Insomnia Management Flow chart" to be used at admission point, training sessions for ward staff, shared teaching programmes with patients at their sleep management sessions, face to face and email correspondence to inform medical trainees about this project and gathering feedback from patients and staff before and after this project. 
Result. The results of this project demonstrated a total reduction in hypnotic tablet administration was very significant with a $44.5 \%$ reduction post intervention.

Conclusion. This demonstrates the positive change in our clinical practice that has resulted from our interventions. This will improve patient safety and reduce cost of hypnotic medications for the NHS. Following on from this initial intervention, we feel that we can continue to make further changes and expand the changes we made on this ward, to other similar wards in our hospital, trust and to other inpatient psychiatric wards further afield.

\section{A qualitative study of foundation year two (F2) doctor's attitudes towards psychiatry carried out in Northern Ireland}

Michael Doris $^{1 \star}$, Kathyrn Mitchell ${ }^{1}$, Damien Hughes ${ }^{1}$, Lorraine Parks ${ }^{2}$ and Angela Carragher ${ }^{2}$

${ }^{1}$ Belfast HSC Trust and ${ }^{2}$ South Eastern Health and Social Care Trust ${ }^{*}$ Corresponding author.

doi: 10.1192/bjo.2021.111

Aims. Recruitment into psychiatry is a major issue nationally. Northern Ireland (NI) consistently punching above it's weight with psychiatry recruitment - in a region that only attracts $31.8 \%$ of F2s to enter into any training programme, Core psychiatry has been consistently oversubscribed. Here we look to examine the experiences of F2s in NI, including those who have had a placement in psychiatry and those who have not - what can we learn from NI? Background. The exposure to psychiatry during the F2 year is a crucial time for recruitment to psychiatry. In NI, where there has been an $100 \%$ fill rate at core training level for many years, trainees and consultants have pointed towards a positive experience in the F2 year.

Method. Questionnaires were given out at a sample of F2 Generic Skills sessions, gathering a range of quantitative and qualitative data. A representative sample of over half of current F2s wrote about there preconceptions and experiences of psychiatry, whether they had worked in it or not. An a priori approach was taken towards generating codes as part of a framework analysis from which 4 major themes were identified.

Result. 93/148 F2 doctors who were approached responded to the survey of which $36.6 \%$ had experienced a Foundation placement in psychiatry. Major qualitative themes that emerged were exposure to psychiatry, the nature of working in psychiatry, being valued and stigma. Doctors who had an F2 placement were much more likely to be willing to pursue a career in it, regardless of whether they had been allocated a placement with psychiatry by choice or not.

Conclusion. This survey adds to the literature that exposure to psychiatry in undergraduate and postgraduate level has a huge role in shaping attitudes towards the specialty of psychiatry, and indeed the likelihood of a foundation doctor going on to become a psychiatry trainee. Stigma in the medical profession towards mental illness and psychiatry remains prevalent.

\section{Benefits observed in an inpatient MDT programme} for FND are not associated with medication use or previous therapies

\author{
Thomas Elliott ${ }^{1 \star}$ and Michael Elmalem ${ }^{2}$ \\ ${ }^{1}$ Camden and Islington NHS Foundation Trust and ${ }^{2}$ National \\ Hospital for Neurology and Neurosurgery \\ ${ }^{\star}$ Corresponding author.
}

doi: 10.1192/bjo.2021.112
Aims. The National Hospital for Neurology and Neurosurgery provides various services for patients with Functional Neurological Disorder (FND), including a four-week inpatient rehabilitation programme run by an integrated Multi-Disciplinary Team (MDT) of Occupational Therapists (OT), Physiotherapists (PT), Psychologists and Psychiatrists.

We had observed that patients with FND often have medical and psychiatric comorbidities including affective, dissociative, somatic symptom and pain disorders; pharmacological treatments are commonly used. We hypothesised that a high burden of medication, particularly of those which produce dependence, might limit one's ability to entrain therapeutic strategies and therefore benefit from treatment. We additionally hypothesised that patients who had previously tried individual physical or psychological therapies might gain less than those who were treatment-naïve.

Method. In this service evaluation project, we reviewed records from 97 consecutive elective inpatient admissions, comprising the entire intake for 2019 and 2020. Data were extracted from the inpatient discharge summary and therapies discharge report of each patient. We recorded which therapies for FND patients had previously tried (OT; PT; Speech and Language Therapy; Psychology; Pain Service) and the classes of medications they were taking on admission (opiates; benzodiazepines; antidepressants; mood stabilisers; antipsychotics; gabapentinoids). We compared the differentials in outcome measures recorded on the first and last day, including the Canadian Occupational Performance Measure $(n=79)$ and EQ-5D-5L $(n=79)$. Statistical tests of effect size and significance were done using SPSS-25. Group comparisons of EQ-5D-5L were made with Paired t-tests; all other comparisons were done with Wilcoxon signed-rank tests due to non-normal data.

Result. The most common medications used were antidepressants (72\%), gabapentinoids (39\%), opiates (36\%) and benzodiazepines (25\%). $69 \%$ of patients had tried PT, $57 \%$ psychology and $52 \%$ OT, while only $13 \%$ were treatment naïve. Whole-cohort analysis revealed highly significant improvements $(\mathrm{p}<0.001)$ in occupational performance, satisfaction, ratings of general health, subjective difficulty in performing tasks and in pain and fatigue levels. We found no significant differences in outcome measures that correlated with past therapies or medication use.

Conclusion. Our analysis shows that the great majority of our patients gained meaningful benefits from their admission, both on clinician-rated metrics of occupational performance and patient-rated measures of subjective improvement. That there was no significant relationship with therapies or medications suggests, promisingly, that patients taking various medications and with suboptimal responses to previous therapy can still benefit from our MDT programme. Limitations include correlational design, limited generalisability to the general population, missing data for certain outcome measures and the absence of follow-up data.

To resuscitate or not to resuscitate; a question for old age psychiatrists

Liam Embliss* and Mohan Bhat

Goodmayes Hospital, North East London NHS Foundation Trust ${ }^{*}$ Corresponding author.

doi: 10.1192/bjo.2021.113

Aims. The inpatient population of an older adult psychiatric ward will include people with physical and mental health conditions which affect life span and quality of life. Patients may be frail, acutely unwell, or have terminal illnesses such as dementia. It is 